\title{
Photoacoustic imaging of angiogenesis in a subcutaneous islet transplant site in a murine model
}

\section{Wei Shi}

Rena Pawlick

Antonio Bruni

Yasmin Rafiei

Andrew R. Pepper

Boris Gala-Lopez

Min Choi

Andrew Malcolm

Roger J. Zemp

A. M. James Shapiro 


\title{
Photoacoustic imaging of angiogenesis in a subcutaneous islet transplant site in a murine model
}

\author{
Wei Shi, ${ }^{a}$ Rena Pawlick, ${ }^{b}$ Antonio Bruni, ${ }^{\mathrm{b}, \mathrm{c}}$ Yasmin Rafiei, ${ }^{\mathrm{b}}$ Andrew R. Pepper, ${ }^{\mathrm{b}}$ Boris Gala-Lopez, ${ }^{\mathrm{b}, \mathrm{c}}$ Min Choi, ${ }^{\mathrm{a}}$ \\ Andrew Malcolm, ${ }^{b, c}$ Roger J. Zemp, ${ }^{a, *, \dagger}$ and A. M. James Shapiro ${ }^{b, c, t}$ \\ aUniversity of Alberta, Department of Electrical and Computer Engineering, Edmonton T6G 2V4, Canada \\ bUniversity of Alberta, Alberta Diabetes Institute, Edmonton T6G 2C8, Canada \\ 'University of Alberta, Department of Surgery, Edmonton T6G 2C8, Canada
}

\begin{abstract}
Islet transplantation (IT) is an established clinical therapy for select patients with type-1 diabetes. Clinically, the hepatic portal vein serves as the site for IT. Despite numerous advances in clinical IT, limitations remain, including early islet cell loss posttransplant, procedural complications, and the inability to effectively monitor islet grafts. Hence, alternative sites for IT are currently being explored, with the subcutaneous space as one potential option. When left unmodified, the subcutaneous space routinely fails to promote successful islet engraftment. However, when employing the previously developed subcutaneous "deviceless" technique, a favorable microenvironment for islet survival and function is established. In this technique, an angiocatheter was temporarily implanted subcutaneously, which facilitated angiogenesis to promote subsequent islet engraftment. This technique has been employed in preclinical animal models, providing a sufficient means to develop techniques to monitor functional aspects of the graft such as angiogenesis. Here, we utilize photoacoustic imaging to track angiogenesis during the priming of the subcutaneous site by the implanted catheter at 1 to 4 weeks postcatheter. Quantitative analysis on vessel densities shows gradual growth of vasculature in the implant position. These results demonstrate the ability to track angiogenesis, thus facilitating a means to optimize and assess the pretransplant microenvironment. ๑ The Authors. Published by SPIE under a Creative Commons Attribution 3.0 Unported License. Distribution or reproduction of this work in whole or in part requires full attribution of the original publication, including its DOI. [DOI: 10 .1117/1.JBO.21.6.066003]
\end{abstract}

Keywords: photoacoustic imaging; islet transplantation; subcutaneous; device-less.

Paper 160213PR received Apr. 2, 2016; accepted for publication May 9, 2016; published online Jun. 6, 2016.

\section{Introduction}

Diabetes mellitus is a glucose metabolism syndrome characterized by hyperglycemia and glucosuria affecting over 300 million people globally. ${ }^{1,2}$ Type 1 diabetes mellitus (T1DM) is an autoimmune disorder that accounts for $5 \%$ to $10 \%$ of all diabetes cases. T1DM results in the destruction of insulin-producing pancreatic islets of Langerhans leading to uncontrolled glucose homeostasis. ${ }^{3}$ As such, patients afflicted with T1DM require vigilant blood-glucose management and exogenous insulin administration. Islet transplantation (IT) has emerged as a therapeutic clinical treatment for subsets of patients with T1DM, largely owing to the success of the "Edmonton Protocol" by Shapiro and colleagues in 2000. ${ }^{4}$ In clinical IT, cadaveric donor islets are transplanted into the portal vein of the liver, where they subsequently embolize and become vascularized. Despite the clinical success of IT to date, numerous obstacles are associated with the procedure, including, but not limited to, acute islet loss as a result of the instant blood-mediated inflammatory reaction and procedural risks including portal vein thrombosis and bleeding. ${ }^{5,6}$ Additionally, transplantation into the hepatic portal vein limits the ability to image and retrieve donor islets. Bioluminescence imaging was reported for its application in the assessment of pancreatic islet transplanting in the liver and kidney. ${ }^{7}$ However, the quantitative interpretation of bioluminescence imaging was complicated and difficult due to the light

*Address all correspondence to: Roger J. Zemp, E-mail: rzemp@ualberta.ca

${ }^{\dagger}$ Denotes joint senior authorship intensity dependence on varying transfection efficiency of reporter gene inserted to islets, the size of islets, and the parameters used in imaging. As such, there has been an ongoing need to identify a clinically relevant, extra-hepatic transplant site. Such a site would need to be capable of supporting an adequate transplant mass while offering sufficient vascular networks providing oxygen and nutrients during revascularization, thus promoting islet engraftment and function. ${ }^{8}$ Moreover, alternative cellular sources, such as stem cell-derived insulin-producing cells, could be introduced as prospective clinical therapies for T1DM. An attractive option for the transplant of these cell sources is the subcutaneous space, which offers accessibility and retrievability, as well as the potential for biopsy and simple monitoring. ${ }^{6}$ Preclinical IT models investigating the utility of the subcutaneous space as an alternative site for IT have been explored through the use of permanent and temporary devices with varied success. ${ }^{9,10}$ Recently, the Shapiro laboratory established a subcutaneous deviceless (DL) transplant technique (with no need for a permanent encapsulation device) capable of restoring euglycemia in a murine IT model. ${ }^{10}$ In this pivotal study, a hollow nylon catheter was implanted subcutaneously to stimulate a controlled foreign-body response capable of inducing local angiogenesis, thus promoting islet engraftment. The catheter is then removed, and the islets are injected into the prevasculaturized subcutaneous site. This is in contrast to other approaches that sometimes use a porous or mesh bag to host the islets while protecting them against immune attack. Reversal of diabetes without a permanent encapsulation device or exogenous growth factors was demonstrated in Ref. 10. The 
formation of angiogenesis around and within the graft significantly contributed to successful islet engraftment, supplying a densely vascularized cellular graft-supporting matrix for nutrition and physical support. ${ }^{8}$ Inherently, the subcutaneous site is hypoxic, and islets engrafted into the unmodified subcutaneous site fail to routinely reverse diabetes. ${ }^{8}$ In addition to the histological assessment postgraft retrieval, a methodology to monitor such angiogenesis prior to and during islet engraftment is lacking.

Herein, we propose the use of photoacoustic and microultrasound imaging to monitor angiogenesis evoked by a subcutaneously implanted nylon catheter, in longitudinal and nonlongitudinal studies. Photoacoustic imaging has been employed for imaging angiogenesis due to intrinsic high optical contrast between blood and tissue. It is a nondestructive, noninvasive, nonionizing, high contrast, high spatial resolution imaging technology that is an ideal candidate for in vivo applications. In addition, photoacoustic signals are sensitive to hemoglobin concentration and to oxygen saturation due to different optical absorption spectra of oxygenated and deoxygenated hemoglobin. Photoacoustic imaging has been widely used to perform structural imaging of tumor angiogenesis in brains and functional imaging of cerebral hemodynamic changes. ${ }^{11-13}$ Optical-resolution photoacoustic microscopy with micron-level lateral resolution demonstrated its ability to image single capillaries. ${ }^{14,15}$ Using acoustic-resolution photoacoustic microscopy, high resolution at depths beyond the optical transport mean free path and up to a few centimeters can be achieved with diagnostic frequencies or to depths of a few $\mathrm{mm}$ at higher frequencies. To our knowledge, this is the first report on applying photoacoustic imaging to study the angiogenesis induced by temporary implantation of angiocatheters subcutaneously for IT research. The results from the present study may further establish the utility of photoacoustic imaging technology in clinical IT, including the imaging of labeled islets as a means to monitor successful islet engraftment outcomes.

\section{Methods}

A Vevo-LAZR photoacoustic imaging system (FUJIFILM VisualSonics Inc., Toronto, Canada) combining microultrasound and photoacoustic imaging into a common platform was implemented to achieve high frame rate, high-resolution photoacoustic imaging with large field of view, as shown in Fig. 1. A 20-Hz nanosecond flashlamp-pumped Q-switch Nd: YAG laser (680 to $970 \mathrm{~nm}$ ) with optical parametric oscillator and second harmonic generator was used to provide tunable excitation light, which was delivered through optical fiber bundle integrated into a high-frequency 256-element linear array ultrasonic transducer (LZ550, center frequency: $40 \mathrm{MHz}$, axial resolution: $44 \mu \mathrm{m}$, field of view: $14 \mathrm{~mm}$ wide). Multiwavelength excitation is used for further oxygenation studies. Three-dimensional (3-D) imaging was achieved by scanning the array transducer mounted perpendicular to a 3-D acquisition motor, with $\sim 70$ s data acquisition time for $10-\mathrm{mm}$ scanning length at $76 \mu \mathrm{m}$ step size. A heated platform with physiological monitoring capabilities was used to maintain the animal under study as shown in Fig. 1(c).

To create the DL transplant site, a 5-French textured nylon radiopaque angiographic catheter (Torcon $\mathrm{NB}^{\circledR}$ Advantage Beacon ${ }^{\circledR}$ tip Cook Medical, Indiana, USA) was implanted subcutaneously on the left and right ventral surface of the abdomen in mice. At 1,2, 3, and 4 weeks postimplant, catheter recipients were assessed for angiogenesis. Mice were first imaged with catheters in situ and subsequently imaged postcatheter removal. All mice used in our experiment were anesthetized using an anesthesia system integrated with the Vevo-LAZR photoacoustic imaging system. All experimental animal procedures were conducted in conformity with the laboratory animal protocol approved by the Animal Use and Care Committee of the University of Alberta.

In parallel to photoacoustic imaging, tissue samples were harvested subsequent to imaging and histologically assessed for the presence of angiogenesis. Tissue sections were formalin-fixed subsequent to harvest and prepared in paraffin-

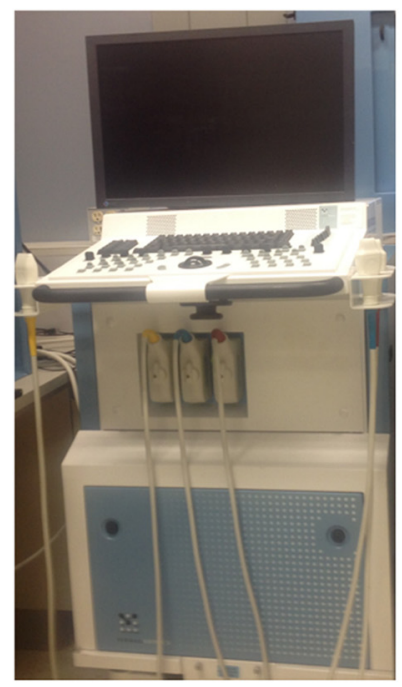

(a)

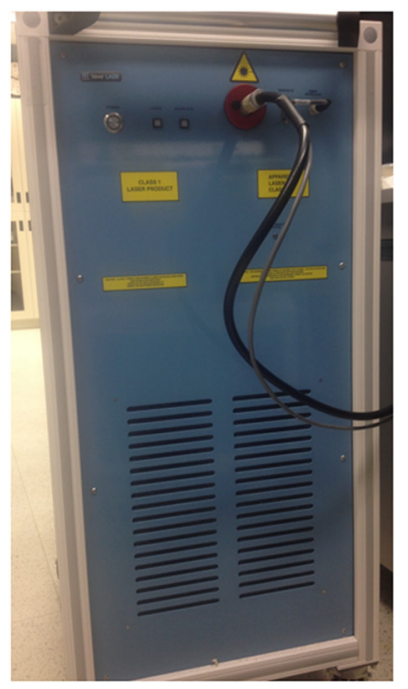

(b)

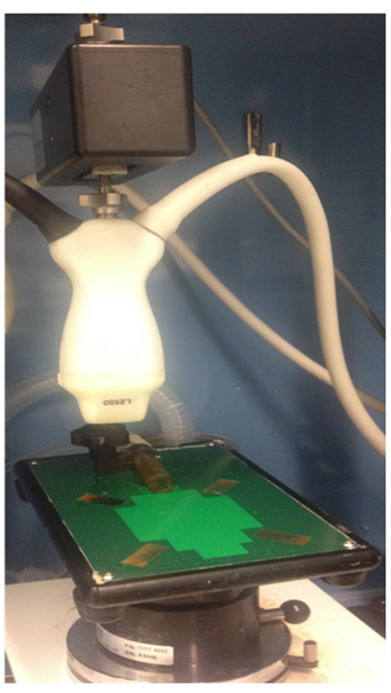

(c)

Fig. 1 Vevo-LAZR photoacoustic imaging system with (a) imaging station, (b) tunable laser, and (c) array ultrasonic transducer integrated with fiber bundle mounted on a 3-D acquisition motor with a heated platform below. 


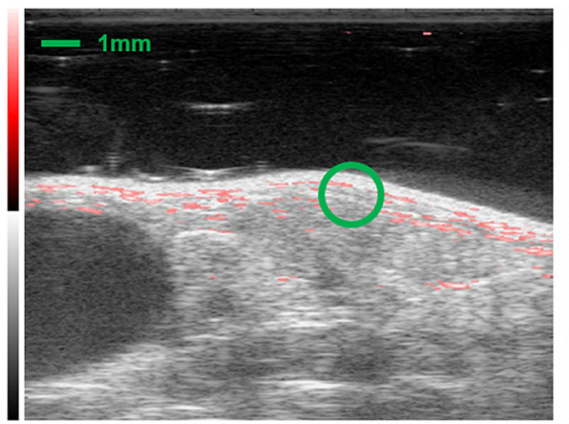

(a)

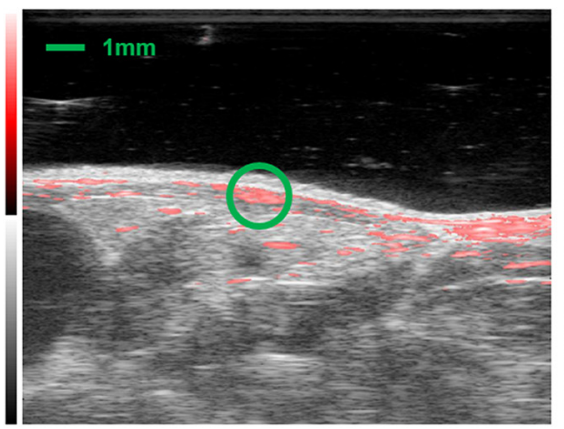

(c)

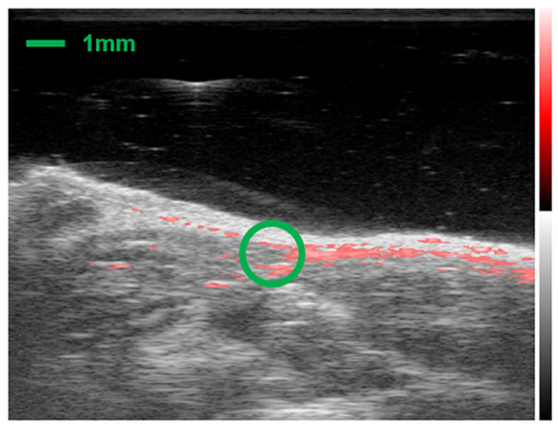

(b)

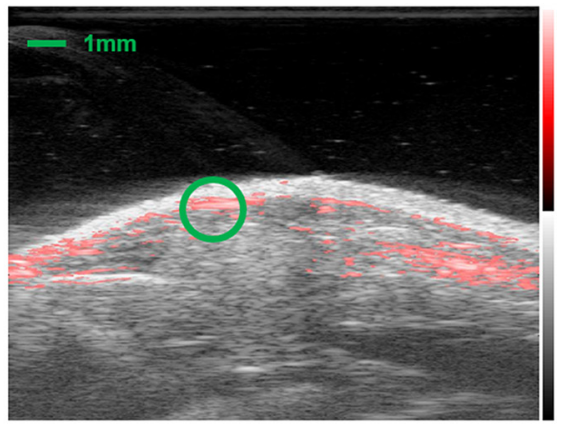

(d)

Fig. 2 Images after catheter removal from mice (a) 1, (b) 2, (c) 3, and (d) 4 weeks postcatheter implantations. Green circle depicts approximate location of catheter prior to removal.

embedded blocks. Identification of endothelial cells for the assessment of vascularization was detected using von Willebrand factor (1:400, Dako A0082) (1:500, Abcam 14935). The sections were also nuclei stained with DAPI (Gibco P36931) followed by the addition of secondary antibodies for immunofluorescence imaging using AxioVision imaging software. Image analysis was completed using ImageJ software (National Institutes of Health, Bethesda, Maryland).

\section{Experiments and Results}

Male BALB/c mice (20 to $25 \mathrm{~g}$ ) were implanted with 2-cm segments of a 5-French textured nylon radiopaque angiographic catheter in the subcutaneous space in the lower left and right abdominal quadrants, parallel to the midline. Four groups of three mice each were implanted with catheters then imaged at different time points after catheter implantation. The different groups were imaged at 1, 2, 3, and 4 weeks, respectively. Images of 3-D were achieved by scanning linear transducer along the direction perpendicular to the implants, where each slide of the two-dimensional image represents a cross-sectional view perpendicular to the longitudinal direction of the implant. Images with catheters in situ were taken first to guide the imaging after the implants were removed, indicating the implant position. Figure 2 shows the images from mice subsequent to catheter removal at 1,2,3, and 4 weeks postimplant. An apparent increase in microvascular density is seen at the implant sites

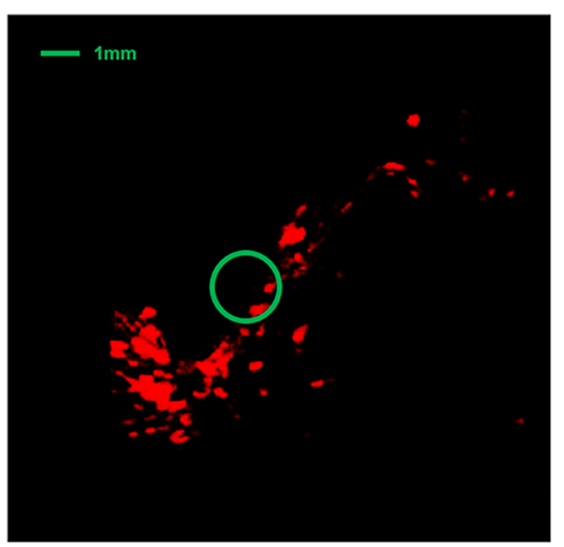

(a)

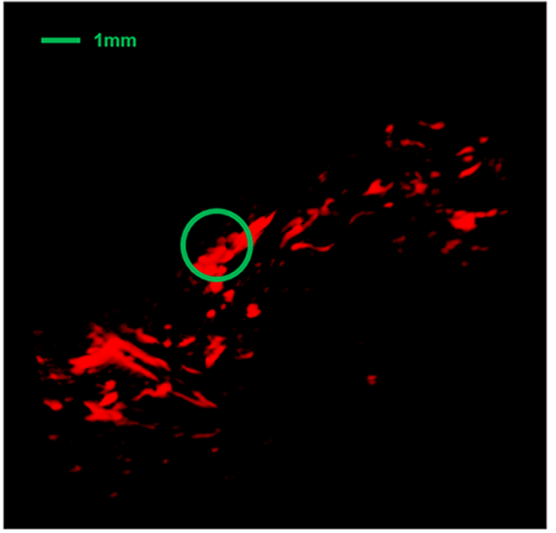

(b)

Fig. 3 Snapshots of 3-D photoacoustic imaging after implants were right removed from mice at (a) 1 and (b) 4 weeks postcatheter implant. Green circle depicts approximate location of the catheter prior to removal. 


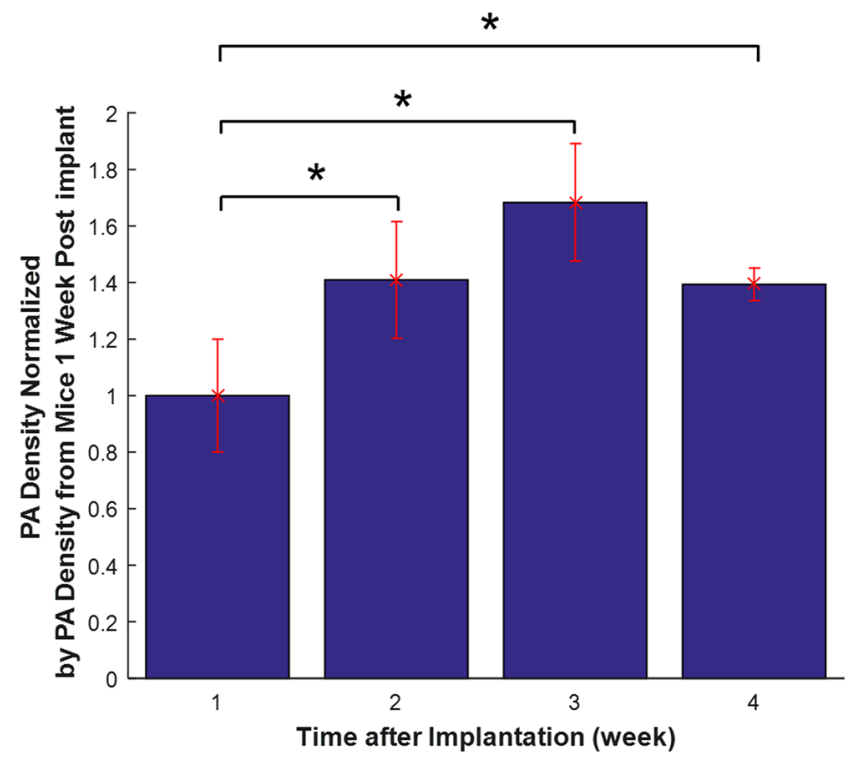

Fig. 4 Quantified PA densities subsequent to implant removal from mice at $1,2,3$, and 4 weeks postcatheter implantation, with 3 mice per week. Data from 2, 3, and 4 weeks postimplant demonstrate significantly greater angiogenesis compared to 1 week postimplantation $(p<0.05)$. PA densities were normalized to PA density obtained from mice 1 week postimplant.

obtained after 2, 3, and 4 weeks of implantation, compared to 1 week of implantation. Increased microvascular density may result in a greater fraction of pixels being occupied by bloodinduced photoacoustic signals but not necessarily stronger signals. Figure 3 shows snapshots from 3-D photoacoustic imaging of mice at 1 and 4 weeks postimplant subsequent to catheter removal. A significantly higher spatial average of reconstructed photoacoustic signals was observed around the implant site in mice after 4 weeks of catheter implantation compared to those from mice after only 1 week of implantation (images from one of three mice per group represented).

Quantitative analysis on vessel densities was conducted using MATLAB programs. The photoacoustic signals from the skin surface were first removed by an algorithm using superimposed ultrasound images. Briefly, pixels at the mouse skin surface were obtained from each A-line ultrasound signal in each B-scan image. A smooth skin profile was then defined using the smooth function and curve-fitting function in MATLAB. Finally, the photoacoustic signals from or above the skin profile were removed. Subsequently, the reconstructed photoacoustic signals were normalized, so each image has the same noise power. To quantify microvascular density, we set the threshold for PA images at $50 \%$ of the maximum and converted pixels above the threshold to a binary 1 , with pixels less than the threshold converted to a binary 0 . Then, we calculated the fraction of pixels with photoacoustic signal (PA density) present inside a $3 \mathrm{~mm} \times 2 \mathrm{~mm} \times 2 \mathrm{~mm}$ volume within the implant sites. Figure 4 shows the PA densities obtained from mice after 1, 2, 3, and 4 weeks postimplantation, normalized to results obtained after 1 week of implantation. These results indicate that vessel growth was significantly greater 2 weeks postcatheter implant. In addition, the statistical studies (ANOVA $F$-test) further demonstrate that the data from 2, 3, and 4 weeks postcatheter implant are significantly greater than the data obtained 1 week postimplantation $(p<0.05)$. Subsequent to photoacoustic imaging, tissue sections were removed and formalin-fixed for histological analysis. Figure 5 shows $10 \times$ images of histological tissue sections harvested after photoacoustic imaging, with Masson's trichrome staining of collagen (blue/green) and evidence of vessel growth (red) along the void area. Immunofluorescent staining of histological preparations revealed increased von Willebrand factor (green) at 3 weeks postimplant, suggestive of angiogenesis (Fig. 6).

\section{Discussion and Conclusions}

Photoacoustic imaging of the modified subcutaneous space using the "deviceless" approach subsequent to 1, 2, 3, and 4 weeks postcatheter implant revealed angiogenesis. Quantitative analysis on vessel densities demonstrated gradual vasculature growth in the implant site as induced by the temporary implantation of the angiocatheter. These results were further supported by histological assessment of the harvested tissue subsequent to photoacoustic imaging, as depicted by Masson's trichrome

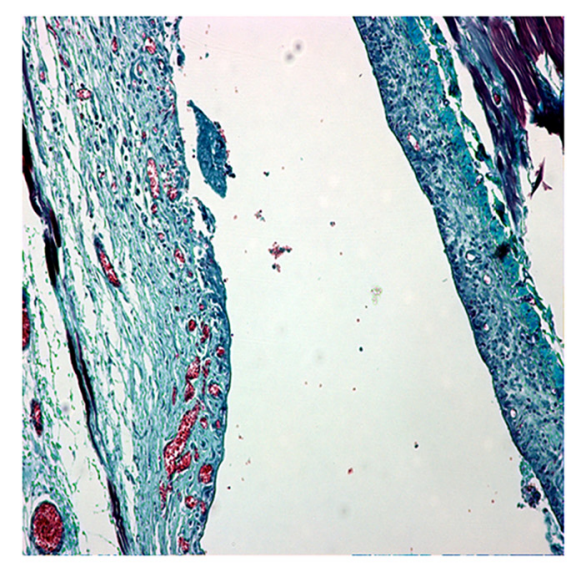

(a)

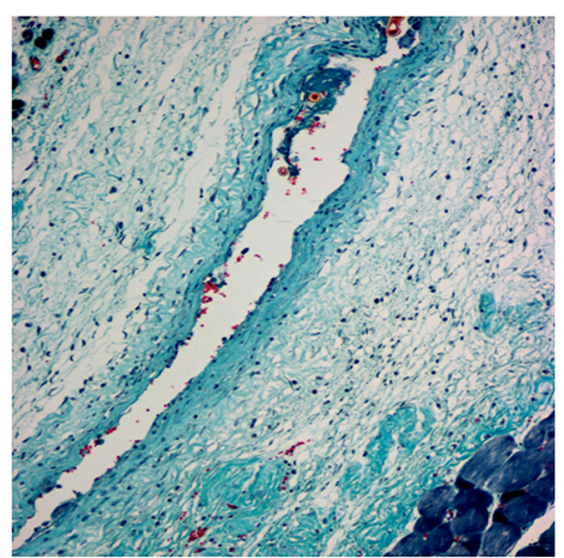

(b)

Fig. 5 Masson's trichrome histological assessment of tissue sections harvested subsequent to photoacoustic imaging. 10x images of tissue from mice at (a) 1 and (b) 3 weeks postcatheter implantation. The presence of blood vessels is depicted by the red staining within the collagen (blue/green) and around the implant sites. 


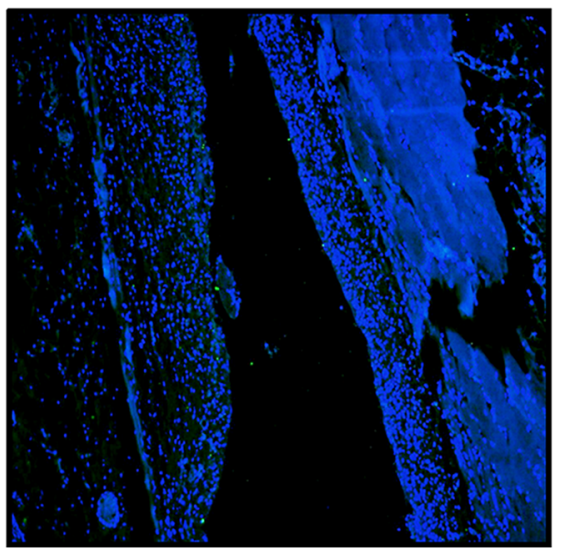

(a)

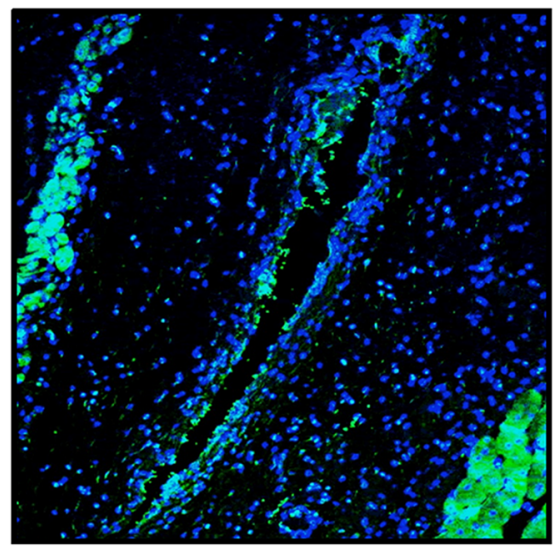

(b)

Fig. 6 Immunofluorescent imaging of tissue sections harvested subsequent to photoacoustic imaging at (a) 1 and (b) 3 weeks postcatheter implantation. The presence of blood vessels is depicted by von Willebrand factor (green) while DAPI-stained nuclei stained positive in blue (10x).

staining and immunofluorescence with von Willebrand factor. The decrease of PA density at 4 weeks postcatheter implantation may have resulted from the catheters shifting from the abdominal site. This may be plausible, as previous studies utilizing the DL subcutaneous exhibited robust vessel growth at 4 week's postcatheter implantation. ${ }^{10}$

To our knowledge, this is the first report utilizing photoacoustic imaging to identify angiogenesis at the modified subcutaneous site prior to IT. The photoacoustic methodology employed in this study may provide an essential tool in the longitudinal monitoring of islets using a noninvasive and safe imaging technique. This imaging technique may also provide an effective means to assess the optimal time of catheter implantation prior to islet engraftment on a patient-to-patient basis if this technique is employed in clinic IT. Further, combined with islet labeling technology, future preclinical studies may shed light on the events associated with islet engraftment outcomes and potentially translate into a feasible clinical application.

\section{Acknowledgments}

Dr. A.M. James Shapiro is supported through a Senior Clinical Scholarship from Alberta Innovates Healthcare Solutions, AIHS CRIO Team Award \#201201154 and holds a Canada Research Chair in Transplantation Surgery and Regenerative Medicine funded through the Government of Canada. Dr. Roger J. Zemp is supported through a Prostate Cancer Canada Movember Discovery Grant, NSERC (355544-2008, 3753402009, and STPGP 396444), the Canadian Cancer Society (CCS 2011-700718), the Canada Foundation for Innovation, Leaders Opportunity Fund (18472), and the Alberta Advanced Education \& Technology, Small Equipment Grants Program (URSI09007SEG). Wei Shi is supported through the Alberta Innovates Technology Futures Postdoctoral Fellowship Program. Antonio Bruni is supported through scholarships from the University of Alberta, the Alberta Diabetes Institute, and the Alberta Diabetes Foundation. Dr. Andrew R. Pepper is supported primarily through an AIHS Postdoctoral Fellowship and a Fellowship from the Juvenile Diabetes Research Foundation Canadian Clinical Trials Network. Dr. Boris Gala-Lopez is supported by an AIHS Clinician Fellowship. Min Choi is supported through a Queen Elizabeth II Scholarship.

\section{References}

1. A. Agarwal and K. L. Brayman, "Update on islet cell transplantation for type 1 diabetes," Semin. Intervent Radiol. 29(2), 90-98 (2012).

2. J. E. Shaw, R. A. Sicree, and P. Z. Zimmet, "Global estimates of the prevalence of diabetes for 2010 and 2030," Diabetes Res. Clin. Pract. 87(1), 4-14 (2010).

3. D. M. Harlan et al., "Immunology of diabetes S. Current advances and travails in islet transplantation," Diabetes 58(10), 2175-2184 (2009).

4. A. M. Shapiro et al., "Islet transplantation in seven patients with type 1 diabetes mellitus using a glucocorticoid-free immunosuppressive regimen," N. Engl. J. Med. 343(4), 230-238 (2000).

5. N. R. Barshes et al., "Transaminitis after pancreatic islet transplantation," J. Am. Coll. Surg. 200(3), 353-361 (2005).

6. A. Bruni et al., "Islet cell transplantation for the treatment of type 1 diabetes: recent advances and future challenges," Diabetes Metab. Syndr. Obes. 7, 211-223 (2014).

7. J. M. Virostko, "Assessment of pancreatic islet transplants using in vivo bioluminescence imaging," Master's Thesis, Vanderbilt University, Nashville, Tennessee, http://etd.library.vanderbilt.edu/available/etd12032003-162124/unrestricted/etd.pdf (2003).

8. A. R. Pepper et al., "Revascularization of transplanted pancreatic islets and role of the transplantation site," Clin. Dev. Immunol. 2013, 352315 (2013).

9. A. R. Pepper et al. "Diabetes is reversed in a murine model by marginal mass syngeneic islet transplantation using a subcutaneous cell pouch device," Transplantation 99(11), 2294-2300 (2015).

10. A. R. Pepper et al., "A prevascularized subcutaneous device-less site for islet and cellular transplantation," Nat. Biotechnol. 33(5), 518-523 (2015).

11. G. Ku et al., "Multiple-bandwidth photoacoustic tomography," Phys. Med. Biol. 49(7), 1329-1338 (2004).

12. G. $\mathrm{Ku}$ et al., "Imaging of tumor angiogenesis in rat brains in vivo by photoacoustic tomography," Appl. Opt. 44(5), 770-775 (2005).

13. X. Wang et al., "Noninvasive laser-induced photoacoustic tomography for structural and functional in vivo imaging of the brain," Nat. Biotechnol. 21(7), 803-806 (2003).

14. K. Maslov et al., "Optical-resolution photoacoustic microscopy for in vivo imaging of single capillaries," Opt. Lett. 33(9), 929-931 (2008).

15. W. Shi et al., "In vivo near-realtime volumetric optical-resolution photoacoustic microscopy using a high-repetition-rate nanosecond fiberlaser," Opt. Express 19(18), 17143-17150 (2011).

Wei Shi is a postdoctoral fellow working in the Department of Electrical and Computer Engineering at the University of Alberta. Her research interests include biomedical optics, biomedical imaging, and fiber optics.

Roger J. Zemp earned his BSc degree in physics from the University of Alberta in 1998, his MASc degree from the Institute of Biomaterials 
and Biomedical Engineering at the University of Toronto in 2000 and his $\mathrm{PhD}$ in biomedical engineering from the University of California Davis in 2004. He worked as a postdoctoral fellow under the supervision of professor Lihong Wang at Texas A\&M University from 2004 2006 then at Washington University in St. Louis from 2006-2007. He is currently an associate professor of electrical and computer engineering at the University of Alberta.

A. M. James Shapiro led the development of the "Edmonton Protocol," and was the lead author on the important The New
England Journal of Medicine paper in 2000. He currently leads a national project in ex vivo organ transplant repair and has active clinical trials in islet and stem cell transplantation. He holds a CRC chair in transplant surgery and regenerative medicine and a clinical senior scholar with AlHS, and is a fellow of the Royal Society of Canada and Canadian Academy of Health Sciences.

Biographies for the other authors are not available. 